\title{
THE LHC TEST STRING: RESULTS FROM RUN 2
}

\author{
R.Saban, E.Blanco-Viñuela, F.Bordry, L.Bottura, D.Bozzini, C.Calzas-Rodriguez, E.Carlier, \\ R.Denz, V.Granata, R.Herzog, Q.King, D.Milani, B.Puccio, F.Rodriguez-Mateos, R.Schmidt, \\ L.Serio, F.Tegenfeldt, H.Thiesen, R.van Weelderen, CERN, Geneva, Switzerland
}

\section{Abstract}

After the commissioning and the first powering of the main circuits in autumn 2001 in its shorter version, the facility was completed to a full cell of LHC in the regular part of an arc and commissioned in July 2002. During this second run, which accumulated more than 4000 hours below $2 \mathrm{~K}$, a very dense experimental program was carried-out to validate the final versions of the technical systems and design choices such as the bus-bar cables running along the magnet cold masses inside the cryostats. The program included the investigation of thermo-hydraulics of quenches, quench propagation, power converter controls and tracking between power converters. The cryogenic process dynamics were studied in length; predictive control techniques were tested and their performance assessed.

During a short shutdown starting in December 2002, the facility was stripped of all instrumentation contributing to increased heat loads and heat load measurements will be performed in a last run during the first half of 2003.

The paper describes the facility and details the results obtained during the experimental program.

\section{THE STRING PROGRAMME}

String 2 [1] was assembled in two phases starting in January 2000. It follows the final design of LHC with the cryogenic helium distribution line running alongside the magnets. During the first phase, the facility, which comprised an electrical feed box, a half-cell and an additional short straight section, was assembled using exclusively prototype components specially instrumented for the experimental programme. The three dipoles, which were added during Phase 2 [2], are machine worthy preseries magnets. During Phase 2, 19 quenches in the dipole circuit were provoked and one training quench was observed. The dipoles were ramped to nominal current $(11850$ A) 15 times. No natural quench was observed in neither of the quadrupole circuits which were powered to nominal current 6 times and quenched 9 times.

\section{VALIDATIONS AND MEASUREMENTS}

\section{Quench Propagation}

The extent of the propagation in LHC-like decay times on a full cell was measured by a decay time constant of $100 \mathrm{~s}$ like for the dipole circuit in an LHC arc; for this, the power converter was programmed to continue supplying an exponentially decaying current after the first magnet was quenched. This was possible because of the robustness of the converter current loop (RST): the time constant during the event varied from $1280 \mathrm{~s}$ to $200 \mathrm{~s}$. The extent of the propagation confirms that it will be limited to a full cell.

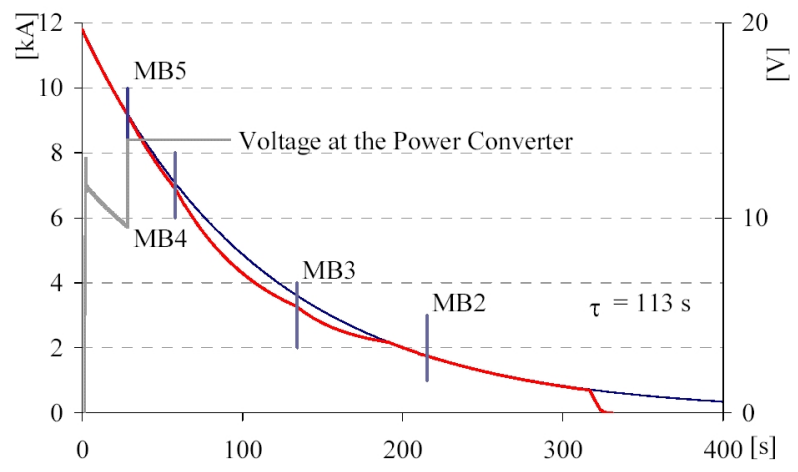

Figure 1: Quench propagation experiment. The quench heaters were fired on the last dipole (MB6) and helium forced towards the neighbouring dipoles. The propagation stops before the last dipole (MB1).

\section{Pressure in the Cold Mass Following a Quench}

The quench recovery procedure using one (out of the two) relief valve confirmed that a complete cell could be discharged within the pressure design limits of the helium vessels.

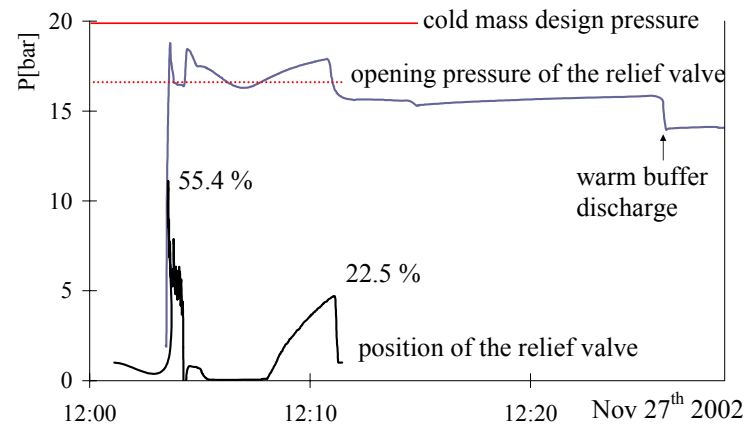

Figure 2: Evolution of the pressure in the cold mass after a full house quench. The quench heaters were fired on all dipole and quadrupole magnets simultaneously.

\section{Cryogenic Process Control}

The highly non-linear nature of the $1.9 \mathrm{~K}$ cooling loop dynamics, which exhibits variable time length delay and inverse response, required the use of a more advanced regulator than the standard PID controller to maintain the magnet temperatures at the desired value.

The tuning of the PID controller was done with the objective of avoiding undesired oscillations and 
instabilities. The PID performance was observed to be inefficient in terms of cancellation and recovery of magnet temperature perturbations from varying heat loads.

The implementation of a hybrid model-based predictive controller (MBPC) with an internal state estimator [3] optimized the heat load effect treatment in both excursion and recovery of the magnet temperatures. The use of an estimator gave additional valuable information on the unmeasured He II mass present in the heat exchanger and the dynamic heat load at any moment. Also, the use of an explicit model of the process allowed the controller to adapt itself to the new heat load condition increasing the robustness of the regulator.

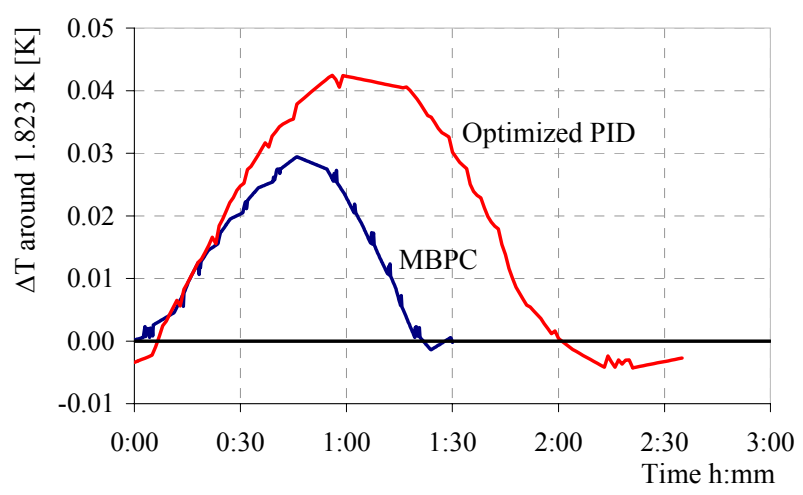

Figure 3: Hybrid model-based predictive controller versus optimized PID control with a $0.2 \mathrm{~W} / \mathrm{m}$ heat load

In Figure 3, the performance of the MBPC controller is compared with an optimized PID when a heat load of 0.2 $\mathrm{W} / \mathrm{m}$ was applied in a step. In both cases the unavoidable temperature excursion is seen but MBPC cancels it earlier and also recovers more rapidly.

\section{Bus bar cables}

One $42 \times 600 \mathrm{~A}$ and three $3 \times 6 \mathrm{kA}$ superconducting cables were installed in a $50 \mathrm{~mm} \varnothing$ tube attached to the shrinking cylinders of the main dipole and quadrupole magnets to evaluate similar configurations in the LHC. There, the cables will conduct current to corrector magnets next to the quadrupoles and to individually powered quadrupoles in the insertion regions.

In a first series of tests the currents in six out of the nine $6 \mathrm{kA}$ conductors and six $600 \mathrm{~A}$ conductors were ramped to nominal current while the voltage on the conductors was continuously monitored for spikes hinting at conductor movement. It was feared that Lorentz forces between conductors in neighbouring cables could lead to conductor movement and subsequent quenches, but during five ramps to maximal current no such event was observed.

Measurements on the expansion of a normal zone after a quench triggered by a spot heater revealed that the behaviour of the cables in the limited space inside the tubes is similar to the behaviour of such cables in a cryostat $[4,5]$. However, transversal quench propagation to conductors in adjacent cables was observed for $6 \mathrm{kA}$ cables, indicating that the heating of a normal zone does substantially change the helium environment in the tube.

The extensive tests (43 provoked quenches) on the installed cables gave us final and positive results for the design, installation and quench protection of the cables in the LHC machine.

\section{EMC of all the components (power converters, quench detection, PLCs, signal conditioners)}

A kicker power supply and a dummy load were installed in the area crowded with power converters, electronics for the interlock system, the quench protection, the control of the switches, the signal conditioning and the cryogenic process control. During a one week run, kicker pulses of varying number and voltage were fired and the performance of the other systems was monitored to detect eventual perturbations. The tests performed with one generator at full voltage in the String represent correctly ( $\mathrm{di} / \mathrm{dt}$ and $\mathrm{dv} / \mathrm{dt}$ ) the operational conditions of 15 generators at injection, which is the most critical situation in terms of signal to noise ratio. This experiment confirmed the electromagnetic compatibility of all the equipment and their interconnections.

\section{Tracking between the three main circuits}

The main result of the tracking measurement campaign is reported in Figure 4 which show the instantaneous value of the ratio $\mathrm{B}_{2} / \mathrm{B}_{1}$ obtained for all runs performed as compared to maximum bounds for the feed-back to lock.

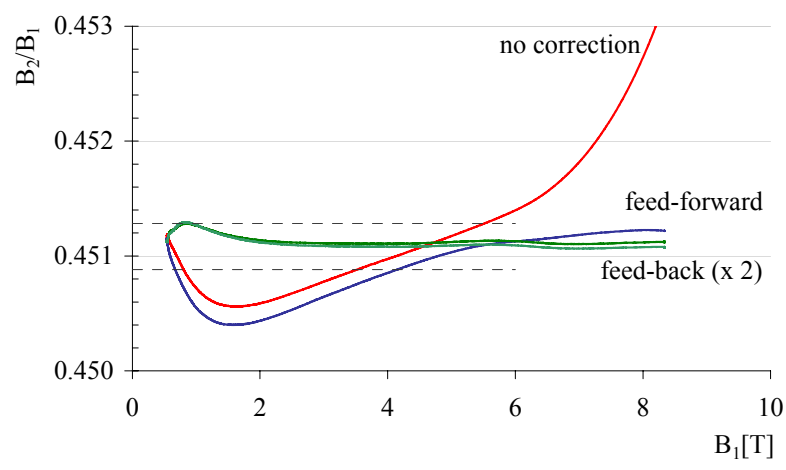

Figure 4: The ratio $B_{2} / B_{1}$ for the focussing quadrupole plotted as a function of the dipole field along the reference current ramp.

The curve marked feed-forward uses the knowledge of the transfer functions as established on the test benches. This correction compensates well the difference in the high field saturation, but leaves a substantial deviation at low and intermediate field. This deviation is mostly due to a systematic measurement artefact. Irrespective of its nature however, and because this is a systematic effect, 
the deviation is well corrected by iteration on the measured $\mathrm{B}_{2} / \mathrm{B}_{1}$ ratio as shown by the curves marked feed-back. In this case the tracking error on the $\mathrm{B}_{2} / \mathrm{B}_{1}$ ratio is definitely inside the range to be achieved for the tune feed-back system to lock, and in fact quite close to the range necessary to maintain the maximum allowed tune variation to within $3 \times 10^{-3}$ as dictated by the nominal LHC performance.

The optimised current ramp was sent unchanged in a second cycle, with the aim to verify the reproducibility of the ramp. The result demonstrates that the reproducibility is excellent.

\section{Quench protection}

String 2 Phase 2 offered the unique occasion to test, for the first time, an LHC like quench protection system on the scale of an LHC full cell. During installation, commissioning and operation a variety of subjects like feasibility of the installation, required functionality as well as reliability and maintainability of the system were critically revised. The outcome confirmed the validity of the basic designs and the functionality of the system. Nevertheless a number of minor problems were identified and already successfully implemented into the latest designs for the LHC quench protection system. For example, the improved designs of quench detection boards and acquisition and monitoring controllers now offer an enhanced functionality and require a significantly lower level of maintenance than those currently installed

The values chosen for thresholds and discrimination time for the main bus-bars, the corrector magnets and their connecting bus bars were sound and only needed little corrections over the commissioning phase. They confirmed the calculations of the quench velocities.

\section{COMMISSIONING}

Procedures, which are expected to become the basis for the commissioning of the LHC sectors, were developed and tested for both the cryogenic system and the electrical system as a whole.

The tests preceding the powering of the circuits $[6,7]$ involved two phases: during the first phase the magnets and the power converters were not electrically connected and were tested separately. The former, involving the magnet side, were aimed at electrical insulation tests at different temperature levels during the cool down. Before the two sides were electrically connected, they were linked together by the interlock system (Powering Interlock System in LHC); the power converters were turned on and tested on a short circuit while the quench protection system was fired to verify the sequence of events. This was repeated for every circuit before the power converter was connected to the current leads on the electrical feed box. While this gradual powering is safe, it proves to be lengthy.
The final tuning of the systems took place following observation of faults induced by the operation in unison with other systems (e.g. false quench signals on the global detectors during converter turn-on and ramp-up).

The optimisation of operation by adapting procedures to particular cases also took place during this phase (e.g. avoiding full quench recovery procedures when auxiliary circuits trip).

The importance of the timing of the commissioning procedures and their dependence on temperature did not go unnoticed: the commissioning of the quench detectors across the superconducting part (few tens $\mathrm{cm}$ ) of a current lead was found to be very tricky (!) after the lead had been cooled down.

A better understanding of the issues related to operating a particular system and observing the effects it can have on other systems took place during this phase. This stimulated the exchange of information between specialists.

\section{CONCLUSIONS}

The String has proven to be a valuable test bed where the validations of the technical systems required before series production and installation could be found. It is the experimental setup where the interplay of the different technical systems could be observed and a variety of measurements carried-out.

It is thanks to the String programme that the actors who will be involved in the hardware commissioning of LHC approach this difficult task with self confidence and enthusiasm.

\section{REFERENCES}

[1] The LHC Prototype Full-Cell: Design Study, LHC Project Report 170, March 1998.

[2] R.Saban et al., First Results and Status of the LHC Test String 2, EPAC '02, June 2002, Paris, France.

[3] E. Blanco et al. Nonlinear Predictive Control in the LHC Accelerator, ADCHEM 03: International Symposium on Advanced Control of Chemical Processes.

[4] R. Herzog, M. Calvi and F. Sonnemann: Quench propagation and heating in the superconducting $600 \mathrm{~A}$ auxiliary busbars of the LHC Advances in Cryogenic Engineering, Vol. 47 p575.

[5] R. Herzog, M. Calvi, F. Sonnemann and J.M. Pelegring-Carcelen: Quench propagation in the superconducting $6 \mathrm{kA}$ flexible busbars of the LHC, Advances in Cryogenic Engineering, Vol. 47 p583.

[6] R.Saban et al., First Powering of the LHC Test String 2, 17th International Conference on Magnet Technology, September 2001, Geneva, Switzerland.

[7] R. Saban, What Have We Learnt from the String Programme (Design, Assembly, Experiments And Measurements)? Workshop on LHC Performance, Chamonix, March 2003. 